\title{
Modelo para avaliação e comparação de projetos de habitação com base no valor
}

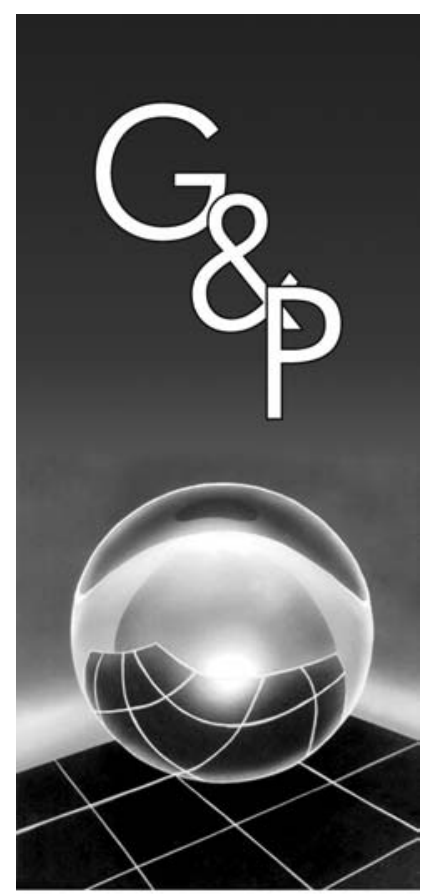

\author{
Adalberto Pandolfo \\ Paulo Maurício Selig \\ Luciana Marcondes Pandolfo \\ Juliana Kurek \\ Luciana Londero Brandli \\ Rafael Lublo
}

\section{Resumo}

Neste trabalho, propõe-se um modelo de avaliação e comparação de projeto de habitação com base no valor, que visa, principalmente, ampliar e melhorar as condições de análise da habitação sob a perspectiva do mercado-alvo. Esse instrumento de avaliação pretende auxiliar o decisor a evitar equívocos que geram perdas em um projeto de empreendimento imobiliário. $O$ desenvolvimento do modelo capacita o tomador de decisão a identificar e mensurar as exigências do usuário de habitação e, ao mesmo tempo, gera informações que podem ser utilizadas durante o processo de projeto da habitação. $O$ modelo proposto baseia-se nas funções percebidas pelo usuário, as quais são representadas pelos atributos da estrutura física, localização e custo, tais como são percebidos pelo mercado-alvo. A sua operacionalização consiste na aplicação de procedimentos que organizam e processam as informações, gerando, como resultado, indicadores de desempenho e perdas provenientes das funções da habitação percebidas pelo usuário e que não agregam valor. Visa com isso melhorar o desenvolvimento de novas concepções de projeto, tornando-o mais atrativo ao mercado-alvo.

Palavras-chave: Avaliação de projetos. Análise do valor. Projeto de habitação.

\section{Introdução}

Com o sistemático acréscimo na concorrência e o aumento da exigência dos usuários, as empresas necessitam cada vez mais aperfeiçoar seu processo de projeto para o desenvolvimento de um novo produto. No setor imobiliário, as mudanças são de tal magnitude que os processos de avaliação de projeto de habitação que não incluem o valor percebido pelo usuário mostram-se incompletos, pois omitem informações vitais para o efetivo auxílio no processo de adequação dos imóveis destinados à habitação.

O objetivo geral deste trabalho é construir um modelo de avaliação e comparação que considere a adequação do projeto de habitação para um determinado público-alvo, por meio de um conjunto de procedimentos, tendo como base o valor percebido pelo usuário. Tem como objetivos específicos: estruturar um procedimento para atender aos usuários de habitação, considerando o valor que eles dão aos atributos percebidos; oferecer uma alternativa de reformulação do projeto que leve à redução de riscos em sua implantação; identificar atributos que têm valor e atributos que não têm valor para o usuário, visando melhorar as condições de competitividade das empresas com relação ao novo projeto; e determinar metas de melhorias dos atributos identificados pelos usuários com baixo valor.

$\mathrm{O}$ modelo proposto busca aperfeiçoar as estratégias de análise do projeto da habitação do ponto de vista do usuário, tornando possível, assim, analisar a cadeia de valor específica para um determinado projeto de habitação. Para aprimorar o projeto, devem-se centralizar os esforços em alguns aspectos no processo de seu desenvolvimento. Um desses aspectos mais valorizados nos estudos atuais é a necessidade de um sistema eficiente de retroalimentação de informações, que permita o aprimoramento progressivo do projeto. 


\section{0 valor da habitação}

O termo valor apresenta um conceito amplo e relativo, de tal forma que as suas interpretações variam em função de sua aplicação. Segundo Pereira Filho (1994), a palavra valor pode apresentar diversos significados, sendo, inclusive, geralmente confundida com palavras custo e preço, diversidade que não é oriunda dos dias atuais. Por volta do ano 350 a.C., Aristóteles já identificava e reconhecia sete tipos de valores: econômico, social, político, jurídico, moral, religioso e estético. Nesta pesquisa, destaca-se o enfoque econômico do valor, por corresponder à menor quantidade monetária para se obter um produto ou serviço que satisfaça precisamente uma função no tempo e com a qualidade necessária.

A análise do valor foi sistematizada no final da década de 1940 pelo norte-americano Lawrence D. Miles, que publicou um trabalho no qual examinava o valor global de um produto na busca de alternativas de menor custo, mantendo as características de desempenho requeridas pelo usuário e identificando desperdícios, pela análise das funções que compõem o produto, o projeto.

A análise do produto por meio da abordagem funcional pode ser definida como a determinação da natureza essencial de uma finalidade, considerando-se que todo objeto, para que exista, destina-se a um fim (CSILLAG, 1995). Desse modo, as funções da habitação devem ser entendidas como o objetivo de seus atributos: a função não é o atributo em si, mas o resultado a ser alcançado por sua existência.

Para Selig (1993), uma função pode ser realizada de diversas maneiras e a importância da Análise do Valor reside no fato de possibilitar que se realize a função da melhor maneira e com o menor custo.

De acordo com Juran (1992), para que se consiga prover o cliente com as funções essenciais de um produto, é preciso estabelecer um processo de relacionamento contínuo entre as funções desempenhadas pelas características do bem e os custos a ele associados.

O tema habitação, segundo Turner (1976), deve ser analisado com base num aspecto de grande importância, qual seja o fato de que constitui a grande base de sustentação para a existência do ser humano. Com esse enfoque, o autor define habitação como a relação de equilíbrio entre três conjuntos de necessidades humanas: abrigo, acesso e ocupação. Salienta, ainda, que o valor da habitação para o usuário é função do que ela pode lhe oferecer e não se restringe apenas à aparência da estrutura física ou à maneira como é vista pela vizinhança.

Nesse sentido, a necessidade de abrigo está vinculada à estrutura física do ambiente construído. A função da habitação é parcialmente atendida enquanto esta estrutura proteger seu morador contra agentes externos e, internamente, proporcionar o atendimento às exigências do usuário.

Com relação ao acesso, verifica-se que o ambiente construído deve estar localizado de forma a propiciar ao morador acesso fácil ao convívio social, às diversas instituições e aos diversos sistemas (água, comunicação, energia e outros). Isso pode ser traduzido pela facilidade de trânsito ao trabalho, às instituições de ensino, à diversão e a outros locais que o usuário deve alcançar na sua rotina de vida.

Há uma crescente busca pela melhoria nos processos de construção, observando-se diversas ações nesse sentido, relacionadas com as padronizações de procedimentos e melhorias do sistema de produção. Contudo, segundo Oliveira (1996), parece que as questões referentes às necessidades, conveniências e expectativas do usuário não têm sido tratadas com a mesma atenção, resultando no desequilíbrio entre os três conjuntos de necessidades apontados por Turner (1976), comprometendo, assim, a qualidade do projeto.

A Análise do Valor percebido pelo usuário tem por objetivo revelar os benefícios desejados da habitação em determinado mercado-alvo e o modo como é percebido o valor relativo das ofertas de imóveis concorrentes. Esta análise está relacionada ao valor que os usuários-alvo atribuem ao projeto de habitação, por meio de seus atributos mais importantes, comparando-os com os projetos de imóveis concorrentes. A determinação do valor percebido é um conjunto complexo de percepções, impressões e sentimentos dos usuários-alvo a respeito do projeto proposto, quando comparado às ofertas de habitações concorrentes. Por isso, é necessária uma abordagem analítica que identifique os componentes dessa percepção e determine sua importância relativa na especificação do valor da habitação para o usuário.

\section{A técnica de Mudge}

Esta técnica implica na comparação das funções e na determinação de sua prioridade relativa. A operacionalização se efetiva pela comparação mútua de todas as funções do produto, a partir da qual se obtém um quadro em que a soma relativa dos coeficientes de cada função representa a importância, em percentual, de uma função sobre a outra (SELIG, 1993).

Segundo Pereira Filho (1994), a utilização desta técnica se baseia na experiência de avaliar o que as pessoas responsáveis pela aplicação dela possuem. Ao se relacionar à sua importância, comparam-se as funções aos pares e é atribuído um fator-peso em razão do grau desta importância. Os fatores-peso utilizados para os graus de importância das funções podem variar de 1 a 5, conforme a definição. Os fatores-peso são avaliações quantitativas da importância relativa durante 
a realização da análise funcional, ao compararem-se as funções duas a duas.

\section{0 modelo proposto}

O modelo é composto por três módulos; cada módulo é constituído por um conjunto de fases que possibilitam definir elementos de avaliação do projeto da habitação com base no valor. Estes módulos são apresentados na Figura 1.

\subsection{Estágio 1 - avaliação do valor percebi- do pelo mercado para os projetos de habitação}

O primeiro estágio avalia um determinado projeto com base no valor percebido pelo mercado. Geram-se indicadores relativos à competitividade do projeto partindo-se das funções percebidas pelos usuários cujo resultado é representado pelos atributos percebidos do imóvel.

\subsubsection{Módulo I - análise do valor percebido pelo mercado}

O Módulo I é formado por oito fases, conforme a Figura 2.

\subsubsection{Fase 1-I: identificação do mercado-alvo e das habitações concorrentes}

O projeto em estudo deve conter informações que possibilitem sua caracterização em relação ao mercado-alvo, bem como a identificação de suas vantagens e desvantagens em relação aos projetos de imóveis concorrentes.

\subsubsection{Fase 2-I: identificação dos atributos percebidos pelo mercado}

\subsection{Atributos da estrutura física e localização}

a) estrutura física: correspondem à área do imóvel, à disposição dos cômodos, à estética e ao material da

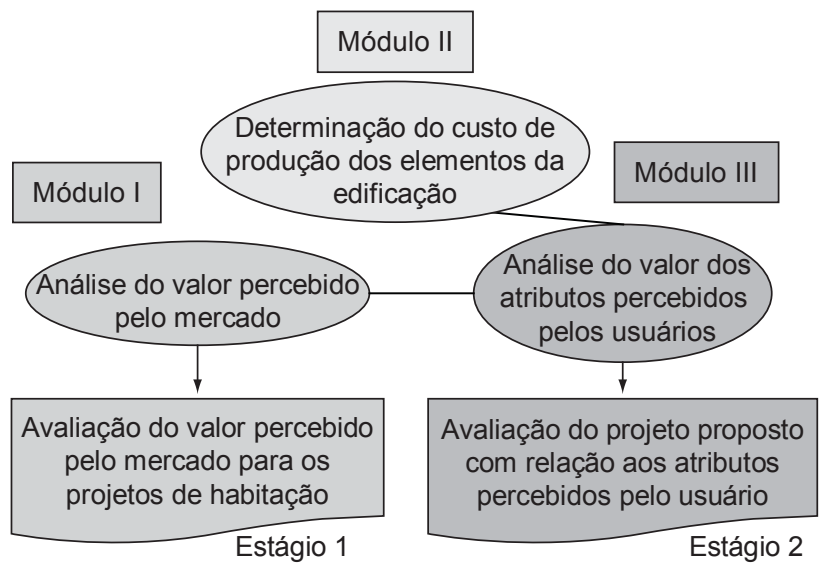

Figura 1. Estrutura de relacionamento dos módulos componentes do modelo de avaliação com base no valor. fachada, à orientação solar, à sacada e aos elementos que propiciam facilidades de uso e segurança para os moradores; e

b) localização: refere-se a aspectos como bairro onde está localizado o imóvel, qualidade do entorno, distância dos locais de comércio e serviços, proximidade com áreas públicas de lazer, clube social e shopping center.

\subsection{Atributos do custo percebido}

Para os atributos do custo percebido, devem-se identificar as características relacionadas com o pagamento pelo uso e manutenção da habitação.

São exemplos: o preço total, o valor de entrada, a taxa de juros do financiamento, o valor das prestações e das parcelas intermediárias.

\subsubsection{Fase 3-I: identificação das funções de- sempenhadas pelos atributos percebidos pelo mercado}

$\mathrm{Na}$ fase anterior, é realizado o levantamento dos atributos do projeto da habitação percebidos pelo mercado. Tais atributos desempenham certas funções que são percebidas pelo usuário e devem ser relacionadas aos respectivos atributos da estrutura física e localização.

\subsubsection{Fase 4-I: determinação da importân- cia relativa dos atributos percebidos pelo mercado}

Solicitam-se a uma amostra do mercado alvo, nos projetos apresentados, os atributos que influenciam na sua decisão de seleção entre alternativas de habitação.

Após a análise dos projetos, solicita-se às pessoas pesquisadas que informem outros fatores que consideram importantes em sua decisão na seleção entre alternativas de habitação e que os projetos avaliados não contemplam. Se existirem necessidades não atendidas, essas devem ser representadas pelos respectivos atributos, os quais serão utilizados, se necessário, na redefinição do projeto em estudo.

Deverá ser determinado um grau de importância relativa também para os atributos não especificados nos projetos, mas considerados necessários pelas pessoas pesquisadas.

Solicita-se às pessoas componentes do mercado-alvo que definam a relação de importância dos atributos identificados nos projetos e, pela técnica de Mudge, se avaliam os vários atributos quanto à sua importância relativa.

Após esse procedimento, aplica-se a técnica de Mudge para os atributos da estrutura física e localização e para os atributos do custo percebido, conforme a Figura 3.

Como resultado, tem-se a posição de importância de cada atributo com relação aos demais, de acordo com a Figura 4. 


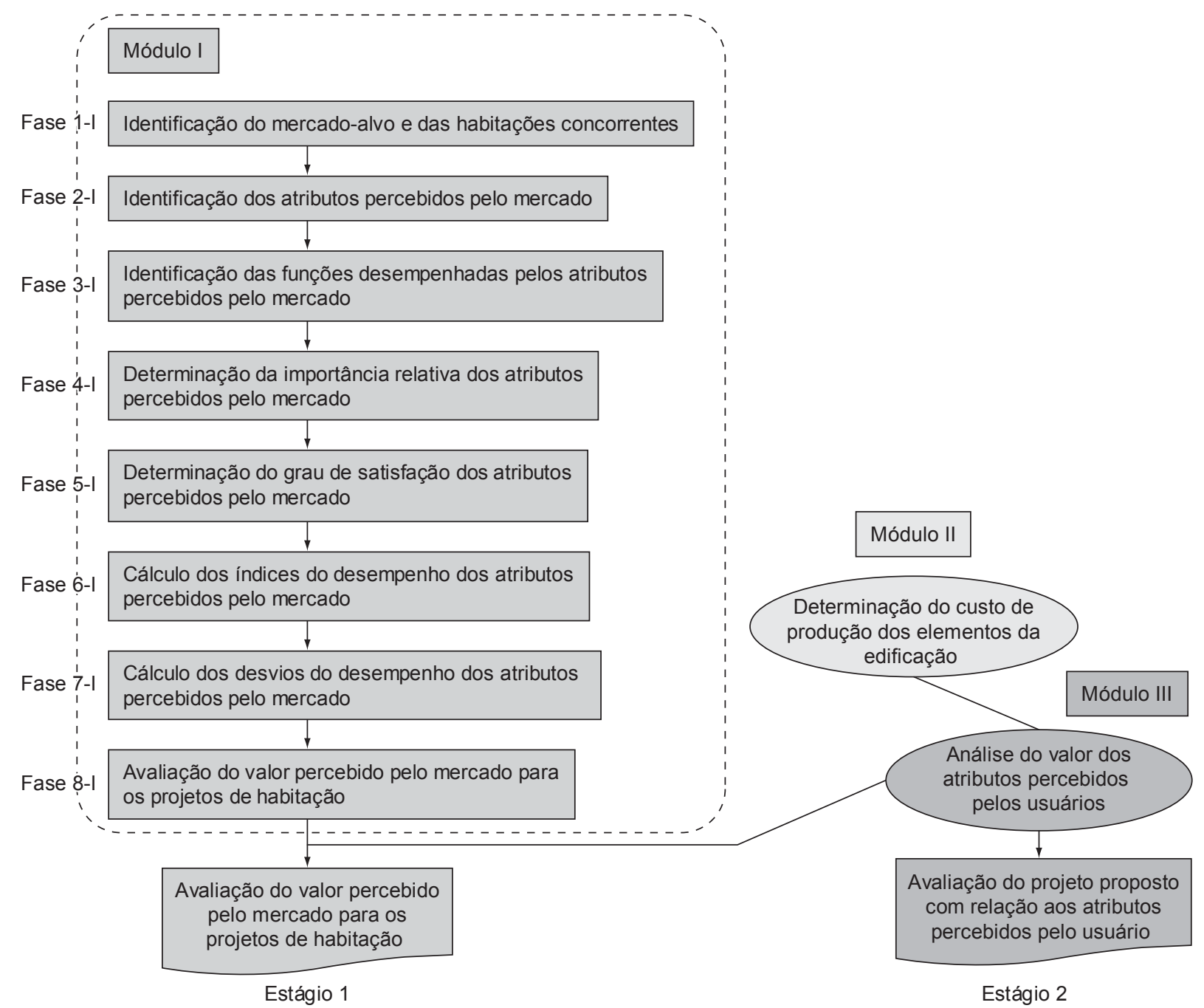

Figura 2. Posicionamento do módulo I na estrutura geral do modelo.

\begin{tabular}{|c|c|c|c|c|c|c|c|}
\hline $\mathbf{8}$ & B & C & D & E & F & Total de pontos & $\begin{array}{c}\text { Importância relativa } \\
\text { (\%) }\end{array}$ \\
\hline A & & & & & & & \\
\hline & B & & & & & & \\
\hline & & C & & & & & \\
\hline & & & D & & & & \\
\hline & & & & E & & & \\
\hline & & & & & F & & \\
\hline & & & & & & & \\
\hline
\end{tabular}

Figura 3. Aplicação da técnica de Mudge para a determinação do grau de importância dos atributos do custo percebido.

\begin{tabular}{|l|l|}
\hline Atributos & Importância relativa (\%) \\
\hline & \\
\hline & \\
\hline & \\
\hline & \\
\hline
\end{tabular}

Figura 4. Importância relativa dos atributos da estrutura física e localização.

\subsubsection{Fase 5-I: determinação do grau de satis- fação em relação aos atributos percebidos pelo mercado}

As pessoas pesquisadas devem atribuir notas que representem sua satisfação em relação a esses atributos (Figura 5). Para tanto, mede-se, inicialmente, o grau de satisfação e, após, inverte-se a escala de variação do nível de satisfação definida pelo grau de importância (Tabela 1).

\subsubsection{Fase 6-I: cálculo dos índices de de- sempenho dos atributos percebidos pelo mercado}

O índice de desempenho dos atributos da estrutura física e localização é determinado utilizando-se a importância relativa e o grau de satisfação. Calcula-se, inicialmente, o desempenho dos atributos por meio da multiplicação da importância relativa pelo grau de satisfação. A soma dessas parcelas tem como resultado o desempenho do projeto com relação aos atributos da estrutura física e localização. 
Por fim, o índice resulta da razão entre o índice de desempenho do projeto e o desempenho médio dos projetos analisados, conforme a Figura 6.

O desempenho dos atributos do custo é obtido pela soma das parcelas que resultam do produto da importância relativa de cada atributo com o respectivo grau de insatisfação.

O índice dos atributos do custo percebido consiste na razão entre o desempenho do custo do projeto e o desempenho médio dos projetos analisados, conforme Figura 7.

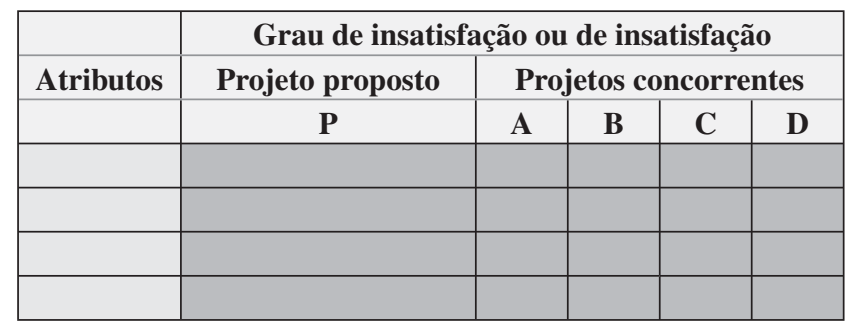

Figura 5. Atribuição do grau de insatisfação em relação aos atributos do custo percebido.

\subsubsection{Fase 7-I: cálculo dos desvios do de- sempenho dos atributos percebidos pelo mercado}

O desvio dos atributos percebidos pelo mercado representa a defasagem de cada atributo do projeto proposto em comparação com os projetos concorrentes. É calculado por meio da diferença em percentual entre o desempenho de cada atributo do projeto proposto e o desempenho dos atributos de cada projeto concorrente.

Tanto o desvio do desempenho dos atributos da estrutura física e localização quanto o desvio do desempenho dos atributos do custo percebido permitem comparar o projeto proposto com os projetos concorrentes.

\subsubsection{Fase 8-I: cálculo do índice do valor da habitação}

O índice do valor da habitação é a razão entre o índice de desempenho da estrutura física e localização e o índice do custo percebido. Assim, o valor percebido aumenta

Tabela 1. Relação entre grau de satisfação e grau de insatisfação em relação aos atributos do custo percebido.

\begin{tabular}{cllc}
\hline $\begin{array}{c}\text { Grau de satisfação com relação aos atributos } \\
\text { do custo percebido }\end{array}$ & \multicolumn{1}{c}{ Nível } & \multicolumn{1}{c}{$\begin{array}{c}\text { Grau de insatisfação com relação aos } \\
\text { atributos do custo percebido }\end{array}$} \\
\cline { 1 - 1 } Escala & & Escala \\
\hline 1 a 2 & Muito insatisfeito & 9 a 8 \\
3 a 4 & Insatisfeito & 7 a 6 \\
5 a 6 & Razoavelmente satisfeito & 5 a 4 \\
7 a 8 & Satisfeito & 3 a 2 \\
9 a 10 & Muito satisfeito & 1 a 0 \\
\hline
\end{tabular}

\begin{tabular}{|c|c|c|c|c|c|c|c|c|c|c|c|}
\hline \multirow{3}{*}{$\begin{array}{l}\text { Atributos da estrutura } \\
\text { física e localização }\end{array}$} & \multirow{3}{*}{$\begin{array}{c}\text { Importância } \\
\text { relativa } \\
(\%)\end{array}$} & \multicolumn{5}{|c|}{ Grau de satisfação com relação aos atributos } & \multicolumn{5}{|c|}{ Desempenho dos atributos } \\
\hline & & \multirow{2}{*}{$\begin{array}{c}\begin{array}{c}\text { Projeto } \\
\text { proposto }\end{array} \\
\mathbf{P}\end{array}$} & \multicolumn{4}{|c|}{ Projetos concorrentes } & \multirow{2}{*}{$\begin{array}{c}\begin{array}{c}\text { Projeto } \\
\text { proposto }\end{array} \\
\mathbf{P}\end{array}$} & \multicolumn{4}{|c|}{ Projetos concorrentes } \\
\hline & & & $\mathbf{A}$ & B & $\mathbf{C}$ & D & & $\mathbf{A}$ & B & $\mathbf{C}$ & D \\
\hline & & & & & & & & & & & \\
\hline & & & & & & & & & & & \\
\hline & & & & & & & & & & & \\
\hline & & & & & & & & & & & \\
\hline Índice do dese & npenho dos atr & utos da est & raf & e lo & açã & & & & & & \\
\hline
\end{tabular}

Figura 6. Matriz das informações para o cálculo do desempenho dos atributos da estrutura física e localização.

\begin{tabular}{|c|c|c|c|c|c|c|c|c|c|c|c|}
\hline \multirow{3}{*}{$\begin{array}{c}\text { Atributos do custo } \\
\text { percebido }\end{array}$} & \multirow{3}{*}{$\begin{array}{c}\text { Importância } \\
\text { relativa } \\
(\%)\end{array}$} & \multicolumn{5}{|c|}{$\begin{array}{l}\text { Grau de insatisfação com } \\
\text { relação aos atributos }\end{array}$} & \multicolumn{5}{|c|}{ Desempenho dos atributos } \\
\hline & & \multirow{2}{*}{$\begin{array}{c}\begin{array}{c}\text { Projeto } \\
\text { proposto }\end{array} \\
\mathbf{P} \\
\end{array}$} & \multicolumn{4}{|c|}{ Projetos concorrentes } & \multirow{2}{*}{$\begin{array}{c}\begin{array}{c}\text { Projeto } \\
\text { proposto }\end{array} \\
\mathbf{P}\end{array}$} & \multicolumn{4}{|c|}{ Projetos concorrentes } \\
\hline & & & $\mathbf{A}$ & B & C & D & & $\mathbf{A}$ & B & C & D \\
\hline & & & & & & & & & & & \\
\hline & & & & & & & & & & & \\
\hline & & & & & & & & & & & \\
\hline & & & & & & & & & & & \\
\hline \multicolumn{7}{|c|}{ Índice dos atributos do custo percebido } & & & & & \\
\hline
\end{tabular}

Figura 7. Matriz das informações para o cálculo do índice dos atributos do custo percebido. 
com avaliações melhores de desempenho da estrutura física e da localização e diminui com o aumento do índice do custo percebido.

O diagrama do valor percebido pelo mercado representado na Figura 8 é construído tendo, na coordenada referente ao eixo do $\mathrm{x}$, os índices de desempenho dos atributos da estrutura física e localização e, na coordenada referente ao eixo do y, os índices do custo percebido. A interseção das linhas referentes a esses dois valores ilustra o posicionamento do índice do valor do projeto em estudo e dos imóveis concorrentes. Define-se a linha

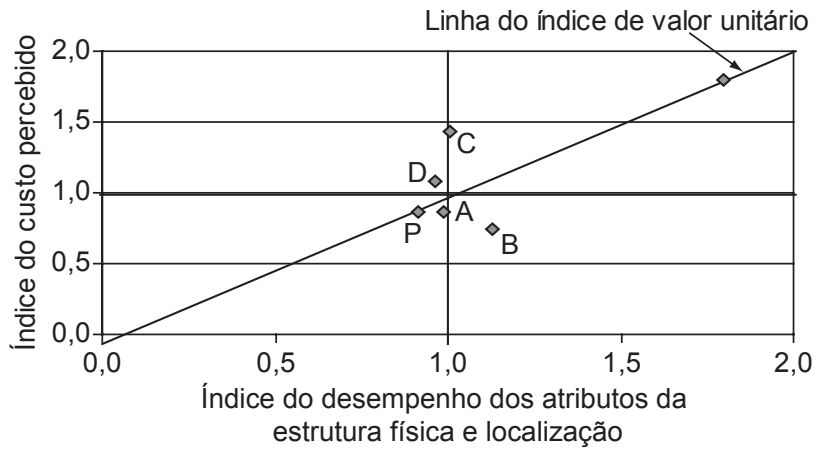

Figura 8. Diagrama do valor percebido pelo mercado. do índice do valor unitário como sendo a reta que corresponde ao conjunto de situações em que o índice do valor da habitação é igual a um, indicando os pontos nos quais existe equilíbrio entre o desempenho dos atributos da estrutura física com os atributos do custo percebido. $\mathrm{O}$ projeto que estiver posicionado abaixo da linha do índice do valor unitário possui maior valor percebido.

\subsection{Estágio 2 - avaliação do projeto proposto com relação aos atributos percebidos pe- los usuários}

A seguir, apresentam-se os módulos II e III, nos quais se determinam o custo de produção dos elementos da edificação e as metas de melhorias dos atributos com valor crítico (Figura 9).

\subsubsection{Módulo II - Determinação do custo de produção dos elementos da edificação}

\subsubsection{Fase 1-II: identificação da estrutura de agregação das partes da edificação}

Nesta fase, inicia-se a análise do projeto da edificação, identificando-se os níveis hierárquicos de sua estrutura de agregação, conforme se observa na Figura 10.

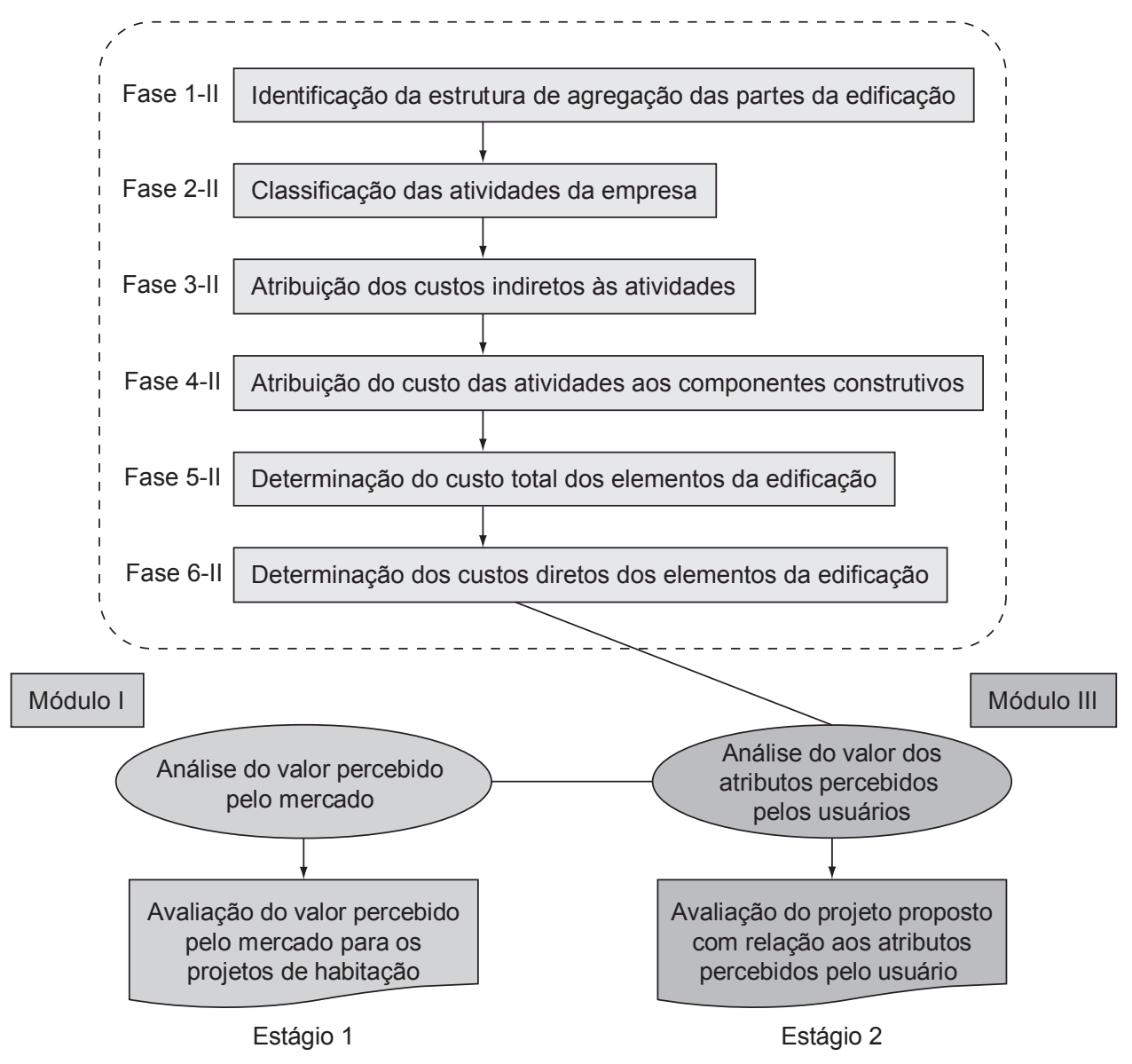

Figura 9. Representação das fases do módulo II e sua ligação com o módulo III na estrutura geral do modelo. 


\subsubsection{Fase 2-II: classificação das atividades da empresa}

As empresas de construção e incorporação possuem um conjunto de atividades distribuídas entre suas diversas funções classificadas em produção e preparação, transporte, atividades auxiliares e apoio administrativo.

\subsubsection{Fase 3-II: determinação dos custos dire- tos dos elementos da edificação}

O custo direto estimado dos elementos da edificação é formado pelo custo estimado de matéria-prima e pela remuneração estimada da mão-de-obra direta de cada um de seus componentes construtivos.

\subsubsection{Fase 4-II: atribuição dos custos indire- tos às atividades}

Com a definição dos itens de custos indiretos, listam-se seus respectivos direcionadores de recursos. Assim, com a definição dos direcionadores de recursos, atribuem-se os custos dos recursos às respectivas atividades.

\subsubsection{Fase 5-II: atribuição dos custos das ati- vidades aos componentes construtivos}

Depois de estabelecidos os direcionadores de atividades, determina-se a capacidade máxima de utilização da atividade pela definição da quantidade de transações que é possível se executarem em um determinado período de tempo.

\subsubsection{Fase 6-II: determinação do custo total dos elementos da edificação}

O custo unitário de produção do elemento da edificação é obtido por meio da soma do custo direto e do custo indireto atribuído aos seus componentes construtivos, conforme apresentado na Figura 11.

\subsection{Módulo III - análise do valor dos atributos percebidos pelos usuários}

O módulo III é formado por cinco fases, conforme a Figura 12.

\subsubsection{Fase 1-III: determinação dos atributos percebidos pelos usuários}

Para o desenvolvimento da primeira fase do módulo III, utilizam-se as informações de saída do módulo I, selecionando-se os atributos da estrutura física, aos quais é possível atribuir custo de produção, conforme Figura 13. Denominam-se estes de atributos percebidos pelos usuários.

\subsubsection{Fase 2-III: determinação da ponderação do desempenho dos atributos percebidos pelos usuários}

A Figura 14 representa a origem das informações para calcular o desempenho dos atributos (matriz de informações geradas no módulo I).

Esta fase compreende três etapas:

a) determinação da importância relativa ajustada dos atributos percebidos pelos usuários;

- a importância relativa ajustada é calculada tendo como referência o percentual de importância relativa definida na Fase 4-I, é determinada por meio da divisão da importância relativa de cada atributo da estrutura física e da localização pelo somatório da importância relativa dos atributos que estão sendo avaliados, conforme a Figura 15; e

b) determinação do grau de satisfação em relação aos atributos percebidos pelos usuários; e

- a determinação do grau de satisfação em relação aos atributos percebidos pelos usuários é obtida a partir das notas de satisfação atribuídas pelas pessoas pesquisadas e tem por base o procedimento desenvolvido na Fase 5-I (Figura 16); e

c) cálculo da ponderação do desempenho dos atributos percebidos pelos usuários

- o desempenho dos atributos percebidos pelos usuários é calculado por meio do produto da importância

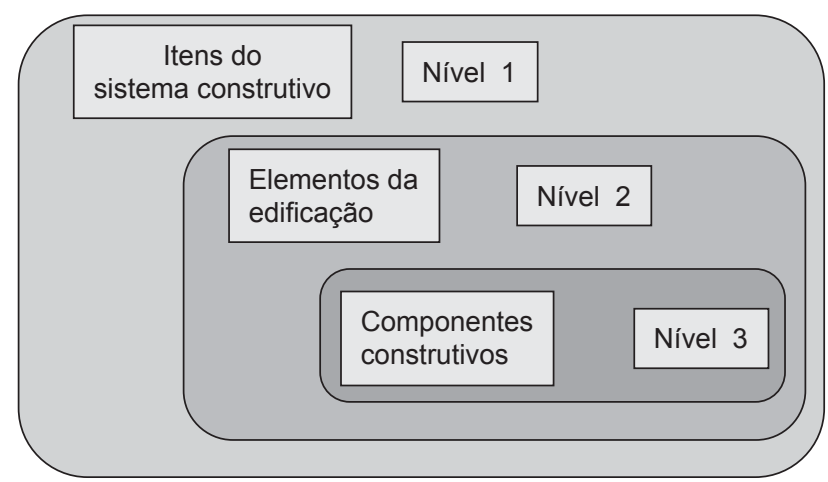

Figura 10. Representação da estrutura de agregação das partes da edificação.

\begin{tabular}{|c|c|c|c|c|c|}
\hline \multicolumn{5}{|c|}{ Elemento da edificação: } & Total \\
\hline Custos & $\begin{array}{c}\text { Componente } \\
\text { construtivo 1 }\end{array}$ & $\begin{array}{c}\text { Componente } \\
\text { construtivo 2 }\end{array}$ & $\begin{array}{c}\text { Componente } \\
\text { construtivo 3 }\end{array}$ & $\begin{array}{c}\text { Componente } \\
\text { construtivo 4 }\end{array}$ & \\
\hline Custos diretos & & & & & \\
\hline Custos indiretos & & & & & \\
\hline Custo unitário de produção & & & & & \\
\hline
\end{tabular}

Figura 11. Custo unitário de produção do elemento da edificação. 


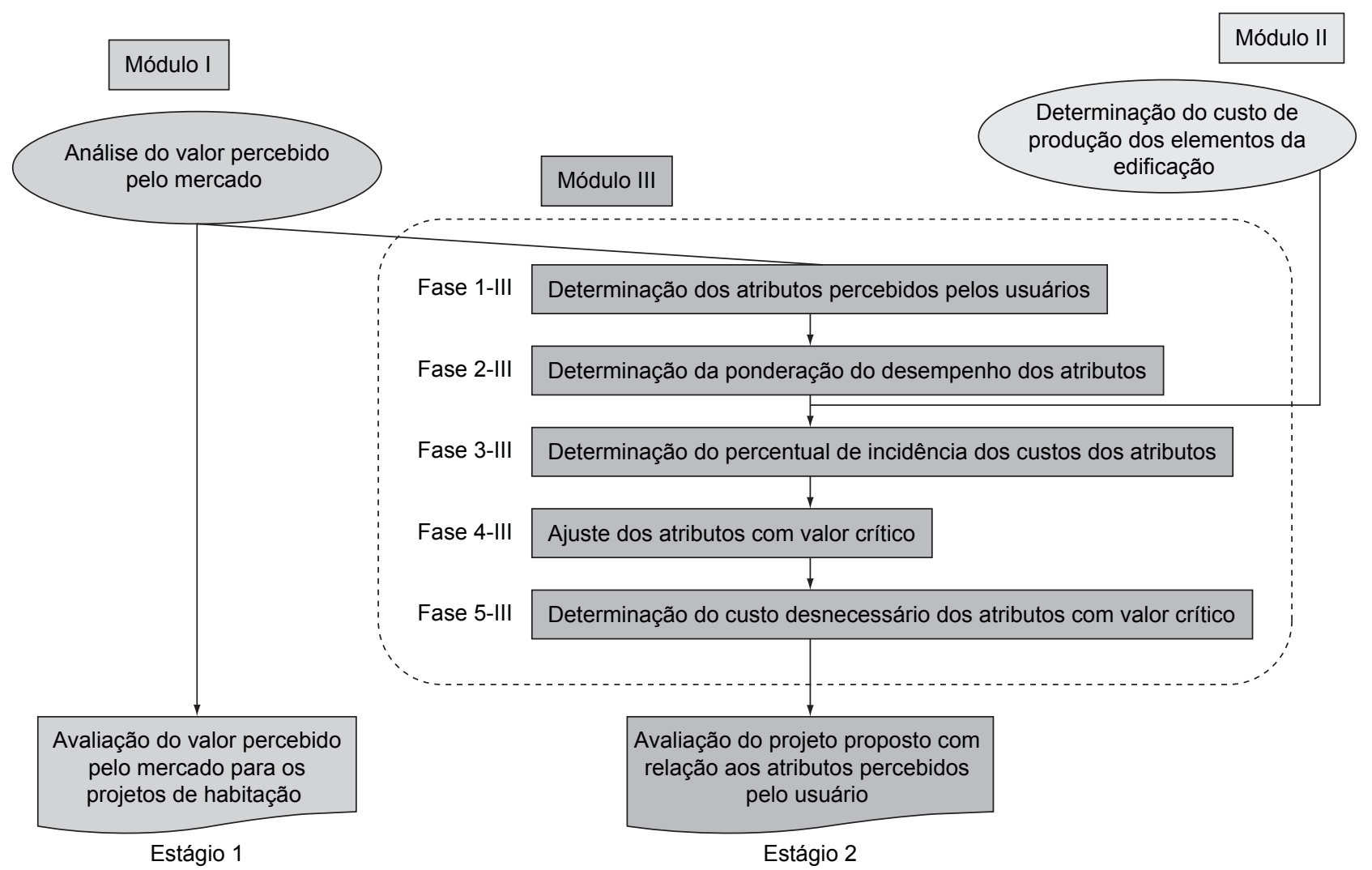

Figura 12. Representação da sequiência de fases do módulo III na estrutura geral do modelo.

Fase 2-I
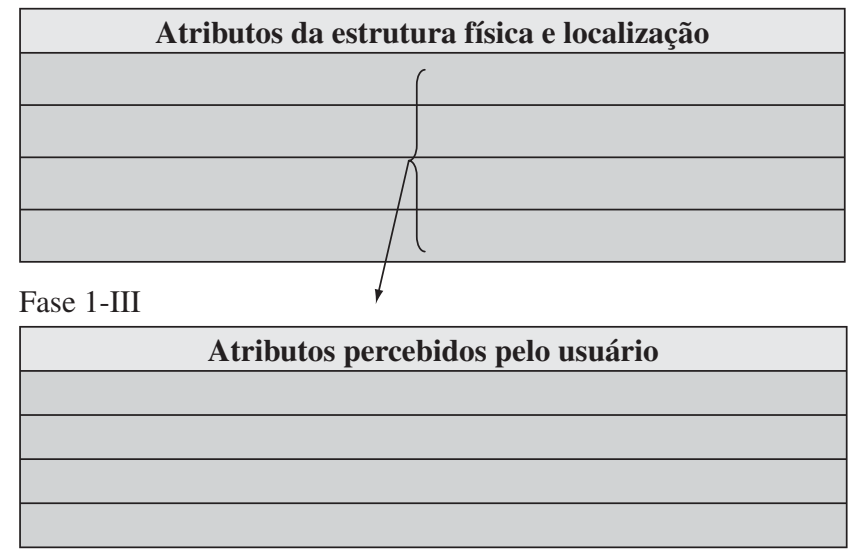

Figura 13. Listagem dos atributos percebidos pelos usuários.

relativa ajustada pelo grau de satisfação em relação a cada atributo. Por fim, calcula-se a ponderação do desempenho dos atributos por meio da razão do desempenho do atributo pelo desempenho total dos atributos avaliados, conforme pode ser visto na Figura 17.

\subsubsection{Fase 3- III: determinação do percentual de incidência do custo dos atributos}

Para calcular o custo do atributo, faz-se o produto do custo de produção unitário do elemento da edificação pela

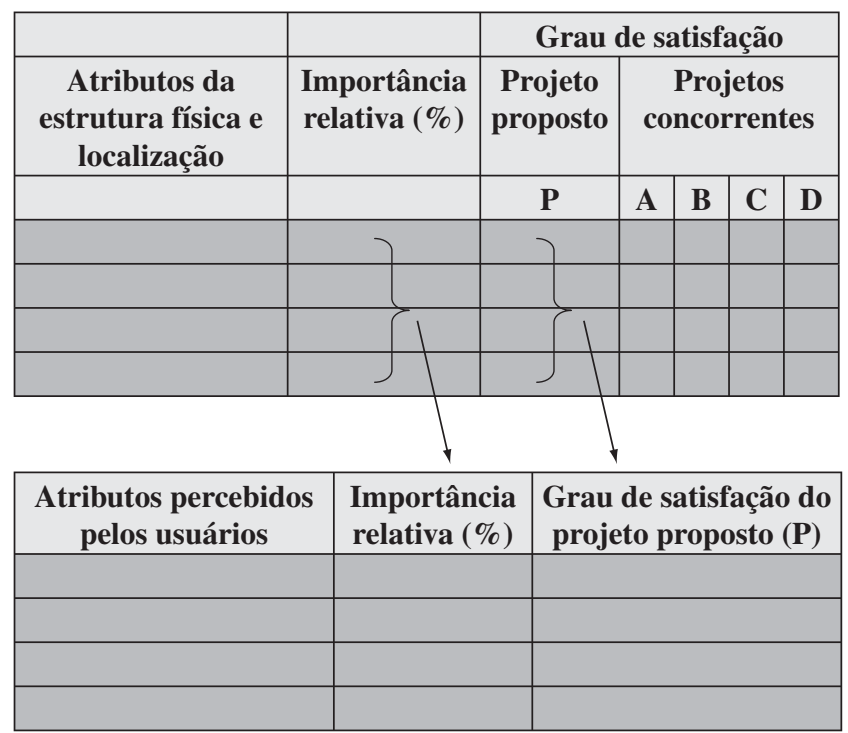

Figura 14. Origem das informações que serão utilizadas para a determinação dos atributos percebidos pelos usuários.

quantidade requerida deste elemento; a seguir, soma-se o custo de produção dos elementos da edificação.

Relacionando-se o custo de produção do atributo percebido pelo usuário com o custo total dos atributos analisados, obtém-se a proporção que este representa no custo total, determinando-se o percentual da incidência do custo de cada atributo. 


\subsubsection{Fase 4-III: ajuste dos atributos com valor crítico}

Esta fase é realizada por meio da análise do índice do valor dos atributos percebidos pelo usuário, relacionando seus desempenhos com seus custos. Descrevem-se abaixo o procedimento de cálculo a ser seguido.

Fase 4-I

\begin{tabular}{|c|c|}
\hline $\begin{array}{c}\text { Atributos da estrutura } \\
\text { física e localização }\end{array}$ & Importância relativa (\%) \\
\hline & \\
\hline & \\
\hline
\end{tabular}

\begin{tabular}{|c|c|c|}
\hline $\begin{array}{c}\text { Atributos percebidos } \\
\text { pelos usuários }\end{array}$ & $\begin{array}{c}\text { Importância } \\
\text { relativa original } \\
(\%)\end{array}$ & $\begin{array}{c}\text { Importância } \\
\text { relativa } \\
\text { ajustada (\%) }\end{array}$ \\
\hline & & \\
\hline & & \\
\hline & & \\
\hline
\end{tabular}

Figura 15. Importância relativa referente aos atributos percebido pelos usuários.

Fase 5-I

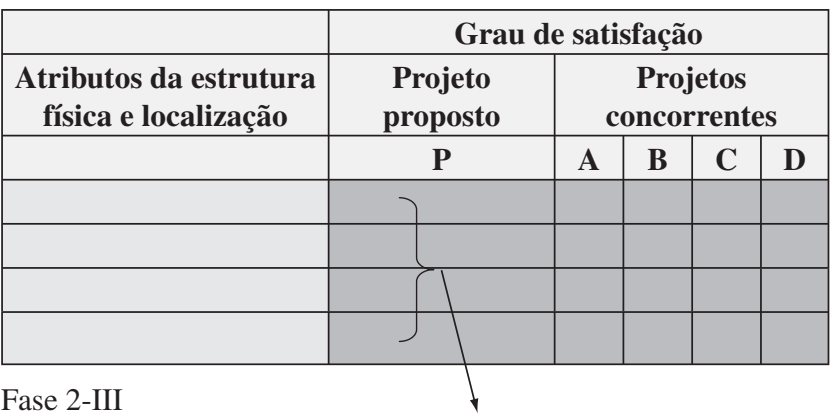

\begin{tabular}{|c|c|}
\hline $\begin{array}{c}\text { Atributos percebidos } \\
\text { pelos usuários }\end{array}$ & $\begin{array}{c}\text { Grau de satisfação com } \\
\text { relação ao projeto proposto }\end{array}$ \\
\hline & \\
\hline & \\
\hline & \\
\hline
\end{tabular}

Figura 16. Atribuição do grau de satisfação em relação aos atributos percebidos pelos usuários.

\subsubsection{Cálculo do índice do valor dos atributos percebidos pelos usuários}

$\mathrm{O}$ valor dos atributos da habitação percebidos pelos usuários é o resultado da razão entre o desempenho e o custo do atributo.

O índice do valor dos atributos é determinado pela razão entre a ponderação do desempenho dos atributos percebidos pelo usuário e o grau de incidência do custo de cada atributo. Este índice possibilita avaliar como cada atributo se comporta com relação ao valor percebido, permitindo aumentar o valor a partir de melhorias em tais atributos, conforme se observa na Figura 18.

\subsubsection{Ajuste dos atributos com valor crítico}

Analisando-se o índice do valor, identificam-se os atributos com índice menor que 1 , o que indica um desequilíbrio entre o desempenho do atributo e seu custo. A Figura 19 ilustra a relação (a ser obtida) dos atributos com índice do valor crítico.

Para os índices abaixo de um, faz-se necessário reduzir o denominador de tal forma que fiquem iguais ao numerador para que a razão entre estes se torne igual a um. Assim, definem-se equações matemáticas para a determinação do custo desses atributos de tal forma que o índice obtido pela razão do custo do atributo pelo custo total seja igual à ponderação do desempenho do atributo.

Define-se $\mathrm{ca}_{\mathrm{i}}$ - custo do atributo i com índice do valor crítico; na - custo do atributo j com índice do valor não crítico; e da $\mathrm{i}_{\mathrm{i}}$ ponderação do desempenho do atributo $\mathrm{i}$.

Sendo $\mathrm{i}=1,2, \ldots, \mathrm{n} ; \mathrm{j}=1,2, \ldots, \mathrm{m} ; \mathrm{n}=$ total de atributos com índice de valor crítico; e $\mathrm{m}=$ total de atributos com índice de valor não crítico.

Para cada atributo com índice do valor crítico, estabelece-se a relação:

Evidenciando-se ca $\mathrm{i}_{\mathrm{i}}$, obtém-se um sistema de equações lineares com o número de equações iguais ao número de variáveis, conforme apresentado abaixo:

$$
\begin{aligned}
& \mathrm{a}_{11} \mathrm{ca}_{1+} \mathrm{a}_{12} \mathrm{ca}_{2+\ldots+} \mathrm{a}_{1 \mathrm{~m}} \mathrm{ca}_{\mathrm{m}}=0 \\
& a_{22} c_{1+} a_{22} c_{2+\ldots} a_{2 m} c a_{m}=0 \\
& \mathrm{a}_{\mathrm{n} 1} \mathrm{ca}_{1+} \mathrm{a}_{\mathrm{n} 2} \mathrm{ca} \mathrm{a}_{2+\ldots+} \mathrm{a}_{\mathrm{nm}} \mathrm{ca} \mathrm{m}_{\mathrm{m}}=0
\end{aligned}
$$

A resolução desse sistema de equações tem como resultado o custo meta dos atributos.

\begin{tabular}{|c|c|c|c|c|}
\hline $\begin{array}{c}\text { Atributos percebidos } \\
\text { pelos usuários }\end{array}$ & $\begin{array}{c}\text { Importância relativa } \\
\text { ajustada (\%) IRi }\end{array}$ & $\begin{array}{c}\text { Grau de satisfação } \\
\text { GSi }\end{array}$ & $\begin{array}{c}\text { Desempenho dos atributos } \\
\text { DAi = IRixGSi }\end{array}$ & $\begin{array}{c}\text { Ponderação do } \\
\text { desempenho } \\
\text { PDi = DAi/DT }\end{array}$ \\
\hline & IR1 & GS1 & DA1 & PD1 \\
\hline & IR2 & GS2 & DA2 & PD2 \\
\hline & $\ldots$ & $\ldots$ & $\ldots$ & $\ldots$ \\
\hline
\end{tabular}

Figura 17. Relação do desempenho dos atributos percebidos pelos usuários. 


\subsubsection{Fase 5- III: determinação do custo de melhoria dos atributos com valor crítico}

Depois do cálculo do índice do valor, é possível determinar a meta de melhoria de custo no projeto para os atributos que estão sendo avaliados. Para isso, utiliza-se o custo meta de cada atributo, diminuindo-se do custo atual do atributo o custo meta, conforme a Figura 20.

O modelo avalia e compara projetos de habitação, tomando por base o valor atribuído a estes projetos, pelos usuários-alvo. Além de identificar o projeto que mais atende às expectativas do usuário-alvo, também identifica a importância e o grau de satisfação dos atributos percebidos.

\section{Exemplo de aplicação do modelo proposto}

Avalia-se o projeto em estudo, chamado $\mathrm{P}$, em relação a três projetos concorrentes, denominados projetos A, B e C. Os projetos concorrem em um mercado-alvo composto de famílias com até dois filhos e renda mensal entre 2.000,00 e R \$ 3.000,00.

Os imóveis a serem avaliados possuem localização no centro da cidade, tendo como referência o shopping

\begin{tabular}{|c|c|c|c|}
\hline $\begin{array}{c}\text { Atributos percebidos } \\
\text { pelos usuários }\end{array}$ & $\begin{array}{c}\text { Ponderação do } \\
\text { desempenho }\end{array}$ & $\begin{array}{c}\text { Incidência } \\
\text { do custo } \\
(\%)\end{array}$ & $\begin{array}{c}\text { Índice } \\
\text { do valor }\end{array}$ \\
\hline & & & \\
\hline & & & \\
\hline & & & \\
\hline & & & \\
\hline
\end{tabular}

Figura 18. Relação dos índices do valor dos atributos percebidos pelos usuários.

\begin{tabular}{|l|l|}
\hline Atributos percebidos pelos usuários & Índice do valor crítico \\
\hline & \\
\hline & \\
\hline & \\
\hline & \\
\hline
\end{tabular}

Figura 19. Relação dos atributos percebidos com índice do valor crítico.

\begin{tabular}{|c|c|c|c|}
\hline $\begin{array}{c}\text { Atributos } \\
\text { percebidos } \\
\text { pelos usuários }\end{array}$ & $\begin{array}{c}\text { Custo de } \\
\text { produção } \\
\text { atual } \\
\text { (CA) }\end{array}$ & $\begin{array}{c}\text { Custo meta } \\
\text { de produção } \\
\text { (CI) }\end{array}$ & $\begin{array}{c}\text { Meta de melhoria } \\
\text { do custo } \\
\text { (CD = CA - CI) }\end{array}$ \\
\hline & & & \\
\hline & & & \\
\hline & & & \\
\hline
\end{tabular}

Figura 20. Matriz utilizada no cálculo meta de melhoria, dos atributos percebidos pelos usuários. center; são do sexto pavimento, de frente para a rua, todos com dois dormitórios.

Iniciando-se a aplicação pelo Modulo I, realizou-se a análise do projeto e das propostas concorrentes, identificando-se uma lista de atributos que os empreendimentos devem contemplar.

a) estrutura física: corresponde à área do imóvel, à disposição dos cômodos, à estética e material da fachada, à orientação solar, à sacada e aos elementos que propiciam facilidades de uso e segurança para os moradores;

b) localização: refere-se a aspectos como bairro, qualidade do entorno, proximidade do comércio, serviços, áreas públicas e de lazer, clube social e shopping center; e

c) custos percebidos: identificaram-se as características relacionadas com o pagamento pelo uso e manutenção da habitação.

Foi estabelecido um conjunto de informações a serem atendidas pelo projeto $\mathrm{P}$, levando-se em consideração os itens que o caracterizam como concorrente dos demais imóveis (projetos A, B e C) e aplicadas todas as fases componentes do modulo I.

Obtém-se o índice do valor da habitação percebido pelo mercado, que é a razão entre o índice de desempenho da estrutura física e localização e o índice do custo percebido. O índice do valor é representado no gráfico (Figura 21), denominando-se de diagrama do valor.

Analisando-se o diagrama, verifica-se que o projeto $\mathrm{P}$ é o imóvel de maior valor, pois apresenta o maior índice de desempenho e o menor índice do custo. $\mathrm{O}$ projeto em estudo (P) também se situa abaixo da linha do índice do valor unitário e está mais à direita que o projeto do imóvel concorrente B, sendo, portanto, superior a este em termos de valor percebido. Já o imóvel C está acima da linha do índice do valor unitário, apresentando, conseqüentemente, baixo valor percebido pelo mercado.

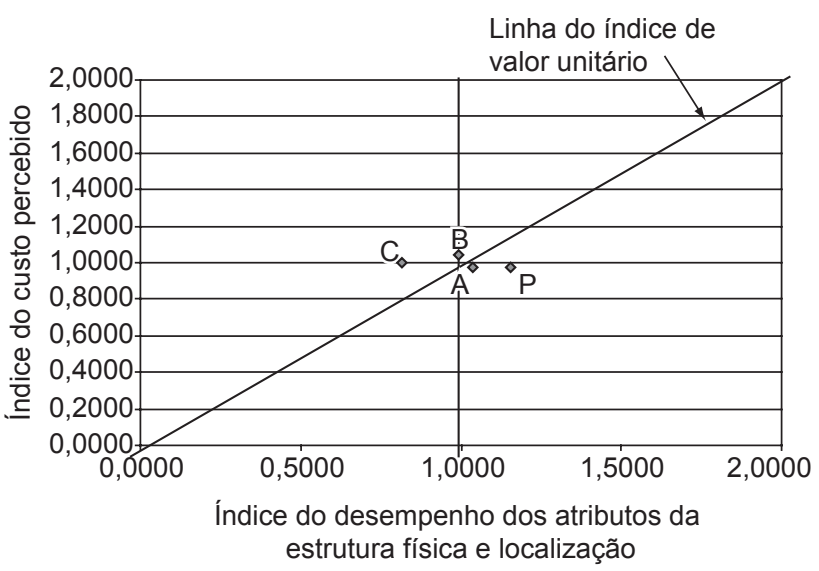

Figura 21. Diagrama do valor percebido pelo mercado para a aplicação com dados reais. 
Após essa análise, são executados os procedimentos do módulo II e módulo III, por meio dos quais se determinam as metas de melhorias dos atributos com valor crítico, proporcionando ajustes nesses atributos.

O índice do valor dos atributos é determinado pela razão entre a ponderação do desempenho dos atributos percebidos pelo usuário e o grau de incidência do custo de cada atributo, conforme apresentado na Tabela 2. Este índice possibilita avaliar como cada atributo se comporta com relação ao valor percebido.

Analisando-se o índice do valor, identificam-se os atributos com índice menor de 1 , indicando um desequilíbrio entre o desempenho do atributo e seu custo, conforme Tabela 3.

A piscina para adulto (A4), o depósito no box da garagem (A5), o banheiro auxiliar (A6) e a sacada (A7) possuem índices do valor abaixo de $1(0,78 ; 0,61 ; 0,74 \mathrm{e}$

Tabela 2. Relação do índice do valor dos atributos percebidos pelos usuários.

\begin{tabular}{lccc}
\hline $\begin{array}{c}\text { Atributos } \\
\text { percebidos pelos } \\
\text { usuários }\end{array}$ & $\begin{array}{c}\text { Ponderação do } \\
\text { desempenho }\end{array}$ & $\begin{array}{c}\text { Incidência do } \\
\text { custo (\%) }\end{array}$ & $\begin{array}{c}\text { Índice do } \\
\text { valor }\end{array}$ \\
\hline $\begin{array}{l}\text { A1 - Estética e } \\
\text { material da }\end{array}$ & 22,25 & 11,00 & 2,02 \\
fachada & & 1,59 & 7,80 \\
$\begin{array}{l}\text { A2 - Persiana nos } \\
\text { dormitórios }\end{array}$ & 12,43 & 24,10 & 1,07 \\
$\begin{array}{l}\text { A3 - Ampla sala } \\
\text { de estar }\end{array}$ & 25,82 & 3,98 & 0,78 \\
$\begin{array}{l}\text { A4 - Piscina para } \\
\text { adultos }\end{array}$ & 3,12 & 10,27 & 0,61 \\
$\begin{array}{l}\text { A5 - Depósito no } \\
\text { box da garagem }\end{array}$ & 6,23 & 15,00 & 0,74 \\
$\begin{array}{l}\text { A6 - Banheiro } \\
\text { auxiliar }\end{array}$ & 11,14 & & \\
$\begin{array}{l}\text { A7 - Sacada } \\
\text { Total }\end{array}$ & 19,01 & 34,05 & 0,56 \\
\hline
\end{tabular}

Tabela 3. Relação dos atributos percebidos com índice do valor crítico.

\begin{tabular}{lc}
\hline Atributos percebidos pelos usuários & Índice do valor \\
\cline { 1 - 1 } A4 - Piscina para adultos & 0,78 \\
A5 - Depósito no box da garagem & 0,61 \\
A6 - Banheiro auxiliar & 0,74 \\
A7 - Sacada & 0,56 \\
\hline
\end{tabular}

0,56). O foco do estudo volta-se, pois, agora, para esses atributos.

Para que o índice do valor seja igual a 1 , o grau de importância e a incidência do custo deverão ser iguais. Assim, estabelecem-se as variáveis C4, C5, C6 e C7, que representam custos menores que os atuais, os quais reduzem os percentuais de incidência, fazendo com que $o$ índice de valor para esses atributos se torne igual a 1.

Para o atributo A4 (piscina para adulto) estabelece-se a razão:

$$
\begin{gathered}
\frac{\mathrm{C} 4}{1.050,00+152,00+2.300,00+\mathrm{C} 4 \mathrm{C} 5+\mathrm{C} 6+\mathrm{C} 7} \\
=0,0312
\end{gathered}
$$

Evidenciando-se C4, C5, C6 e C7, obtém-se:

$$
\begin{gathered}
-0,9684 \mathrm{C} 4+0,0312 \mathrm{C} 5+0,0312 \mathrm{C} 6+ \\
0,0312 \mathrm{C} 7=-109,2624
\end{gathered}
$$

Da mesma forma, para A4, A5, A6 e A7, define-se o sistema de equações lineares:

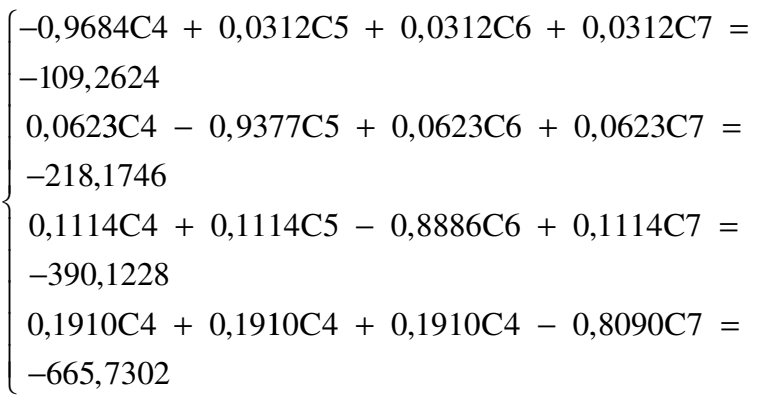

Resolvendo-se este sistema de quatro equações e quatro variáveis, obtêm-se C4, C5, C6 e C7, que são os custos unitários ideais para que o índice do valor seja igual a 1 para a piscina para adulto (A4), o depósito no box da garagem (A5), o banheiro auxiliar (A6) e a sacada do apartamento (A7).

Os custos meta da piscina para adulto, o depósito no box da garagem, o banheiro auxiliar e a sacada do apartamento são, respectivamente, de 180,78; 360,83; 645,22; e $\$ 1.103,11$. Os custos atuais desses atributos são, respectivamente, de 380,00; 980,00; $1.430,00$ e $\$ 3.250,00$. Assim, a meta de melhoria no custo é o resultado da subtração do custo de produção atual e do custo meta de produção, conforme Tabela 4.

Nesta aplicação, constatou-se que o produto deve ser redefinido em alguns aspectos, tornando-se mais atra-

Tabela 4. Meta de melhoria no custo dos atributos percebidos pelos usuários.

\begin{tabular}{lccc}
\hline Atributos percebidos pelos usuários & Custo de produção atual (\$) & Custo meta de produção (\$) & Meta de melhoria no custo (\$) \\
\hline Piscina para adulto & 380,00 & 180,78 & 199,22 \\
Depósito no box da garagem & 980,00 & 360,83 & 619,17 \\
Banheiro auxiliar & $1.432,00$ & 645,22 & 786,78 \\
Sacada do apartamento & $3.250,00$ & $1.103,11$ & $2.146,89$ \\
Total & $6.042,00$ & 2289,94 & $3.752,06$ \\
\hline
\end{tabular}


tivo para o mercado-alvo pela redução de seu custo. Fica evidenciado que os índices do valor menores de 1 indicam um desequilíbrio entre o desempenho do atributo e seu custo; deve-se, assim, reduzir o custo de produção em 52\% na piscina para adultos, em $63 \%$ no depósito do box da garagem, em 55\% no banheiro auxiliar e em $62 \%$ na sacada do apartamento. Isso demonstra que há um considerável desequilíbrio na relação desempenho/ custo desses atributos, a qual deve ser equacionada para que o produto tenha maiores possibilidades de competir no mercado, com preço adequado ao usuário-alvo.

\section{Conclusões}

O modelo apresentado auxilia na elaboração de estratégias competitivas na medida em que se pode obter uma melhor visão de como ocorre a competição no mercadoalvo, pois o modelo de avaliação e comparação proposto leva em consideração os imóveis concorrentes e o desempenho das funções percebidas pelo usuário-alvo, representadas pelos atributos de estrutura física e localização. Trata-se de uma ferramenta para a tomada de decisão no processo de projeto, visto que se fundamenta no atendimento das exigências dos usuários e decorre da avaliação do projeto proposto em comparação aos projetos concorrentes. Sua relevância destaca-se por aumentar as chances de sucesso de um novo produto à medida que demonstra se o projeto é ou não de valor superior.

No exemplo, determinaram-se os atributos que detinham valor crítico, no caso, a piscina para adultos, o depósito no box da garagem, o banheiro auxiliar e a sacada do imóvel. Para cada um desses atributos com valor crítico, calculou-se o custo meta. De posse dessa informação, é possível redefinir os atributos, gerando uma nova proposta de projeto, este, então, mais adequado ao uso pelo mercado-alvo.

Após o término desta aplicação, conclui-se que a avaliação do projeto proposto é necessária, pois destaca os atributos que satisfazem os usuários-alvo, os que não atingem esse objetivo e os que superam as suas expectativas. Os que não atingem o objetivo, pelo alto custo e baixo desempenho (piscina para adultos, por exemplo), devem ser remodelados de forma que, na redefinição do projeto, este contemple as expectativas do usuário.

\title{
Model for the evaluation and comparison of dwelling projects based on value
}

\begin{abstract}
In this work, it is proposed an evaluation and comparison model of dwelling project based on value, which aims, principally, to improve the conditions of dwelling analysis under the perspective of the target market. This evaluation instrument intends to help avoid mistakes which bear unnecessary costs in a real estate business project. The development of the model enables the identification and measurement of satisfaction, expectations and needs of the user of this sector and, at the same time, generates information which may be used during the process of the dwelling project. The proposed model is based on the functions perceived by the user, which are represented by the attributes of the physical structure, localization and cost as defined by the target market. Its operation consists in applying standardized procedures that organize and process the information bearing, as a result, performance indexes and unnecessary costs deriving from the perceived dwelling functions that do not aggregate value. It aims at improving the development of new project concepts making it more appealing to the target market.
\end{abstract}

Keywords: Project evaluation. Value analysis. Housing design.

\section{Referências bibliográficas}

CSILlAG, J. M. Análise do Valor: metodologia do valor: engenharia do valor, gerenciamento do valor, redução de custos, racionalização administrativa. 4. ed. São Paulo: Atlas, 1995.

JURAN, J. M. Juran on quality by design: The new steps for planning quality into goods and services. New York: Free Press, 1992.

OLIVEIRA, R. Habitação ou Construção? Quem dinamiza a economia, como uma proposta da universidade. In:
CONGRESSO TÉCNICO-CIENTÍFICO DE ENGENHARIA CIVIL. V.I., 1996. Anais... Florianópolis, p. 36-41.

PEREIRA FILHO, R. R. Análise do Valor: processo de melhoria contínua. São Paulo: Nobel, 1994.

SELIG, P. M. Gerência e avaliação do valor agregado empresarial. Tese (Doutorado em Engenharia de Produção e Sistemas). Universidade Federal de Santa Catarina, 1993.

TURNER, J. F. Housing of people. London: Marion Boyards, 1976. 


\section{Adalberto Pandolfo}

Programa de Pós-Graduação em Engenharia, Universidade de Passo Fundo,

Campus I, km 171, BR 285, Bairro São José, CP 611, CEP 99001-970, Passo Fundo, RS, Brasil,

e-mail: pandolfo@upf.br

\section{Paulo Maurício Selig}

Programa de Pós-Graduação em Engenharia de Produção, Universidade Federal de Santa Catarina, CP 476, Campus, CEP 88040-900, Trindade, Florianópolis, SC, Brasil,

e-mail: selig @ deps.ufsc.br

\section{Luciana Marcondes Pandolfo}

\section{Juliana Kurek}

Faculdade de Engenharia e Arquitetura, Universidade de Passo Fundo,

Campus I, km 171, BR 285, Bairro São José, CP 611, CEP 99001-970 Passo Fundo, RS, Brasil, e-mail: marcondes@upf.br; jkurek@upf.br

\section{Luciana Londero Brandli}

\section{Rafael Lublo}

Programa de Pós-Graduação em Engenharia, Universidade de Passo Fundo,

Campus I, km 171, BR 285, Bairro São José, CP 611, CEP 99001-970, Passo Fundo, RS, Brasil, e-mail: pandolfo @ upf.br; brandli@upf.br; rafaarq20@ hotmail.com 
